\title{
Flow and Heat Transfer in a Liquid Film over a Permeable Stretching Sheet
}

\author{
R. C. Aziz, ${ }^{1}$ I. Hashim, ${ }^{2}$ and A. K. Alomari ${ }^{3}$ \\ ${ }^{1}$ Faculty of Science and Technology, Open University Malaysia, 50480 Kuala Lumpur, Malaysia \\ ${ }^{2}$ Centre for Modelling and Data Analysis, School of Mathematical Sciences, The National University of Malaysia, 43600 Bangi, Malaysia \\ ${ }^{3}$ Department of Mathematics, Faculty of Science, The Hashemite University, Zarqa 13115, Jordan
}

Correspondence should be addressed to R. C. Aziz; raziana@oum.edu.my

Received 26 November 2012; Accepted 11 December 2012

Academic Editor: Subhas Abel

Copyright $(2013$ R. C. Aziz et al. This is an open access article distributed under the Creative Commons Attribution License, which permits unrestricted use, distribution, and reproduction in any medium, provided the original work is properly cited.

\begin{abstract}
An analysis has been carried out to study the flow and heat transfer in a liquid film over a permeable stretching sheet. Using similarity transformations, the time-dependent boundary layer equations are reduced to a set of nonlinear ordinary differential equations. The resulting parameter problem and velocity as well as temperature fields are solved using the homotopy analysis method (HAM). Analytic series solutions are given, and numerical results for velocity and the temperature profiles are presented through graphs of different values for pertinent parameter. The effects of unsteadiness parameter and permeability parameter on the velocity and temperature profiles are explored for different values of blowing or suction parameter.
\end{abstract}

\section{Introduction}

The study of flow and heat transfer in a thin liquid film is often encountered in industrial and engineering applications. Such processes are wire and fibre coating, cooling of metallic plates, drawing of a polymer sheet, aerodynamic extrusion of plastic sheets, and thinning of copper wires. In polymer processing application, the flow over a permeable stretching sheet has received considerable attention from researchers because of its widespread applications. Therefore, the analysis of momentum and thermal transport within thin liquid film on a continuously stretching surface is important for gaining some fundamental understanding of such processes. Crane [1] studied the Newtonian fluid flow induced by the stretching of an elastic sheet. The boundary layer equation is considered, and the boundary conditions are prescribed at the sheet and on the fluid at infinity. Nonetheless, in real physical application involving coating processes, the consideration of the fluid adhering to the finite liquid film is important. The hydrodynamics of a flow in a thin liquid film driven by an unsteady stretching surface was first investigated by Wang [2] . Andersson et al. [3] explored the heat transfer characteristics of the hydrodynamical problem. Wang [4] investigated the same problem of Andersson et al. [3], presenting analytic solutions. Several researchers extended Wang's [2] classical problem in non-Newtonian problems [5-8], thermocapillarity effects [9-12], and magnetic effects [13, 14].

Wang [4] was probably the first to analyze the flow and heat transfer in a thin liquid film on an unsteady stretching surface using homotopy analysis method (HAM). The HAM is a general analytic method to obtain series solutions for various types of nonlinear equations $[15,16]$. Several studies have successfully applied HAM to various nonlinear problems in science and engineering such as in heat transfer [17], upper-convected Maxwell fluid [18], Kawahara equation [19], diffusion and reaction in porous catalyst [20], micropolar fluid flow [21, 22], and MHD flow [23, 24].

The purpose of the present study is to give a numerical analysis of the flow and heat transfer in a thin liquid film over a porous stretching sheet, and the effects of unsteadiness parameter on the film thickness are explored for different values of blowing or suction parameter. Here the momentum and energy equations are highly nonlinear; hence a similarity transformation is used to transform the nonlinear partial differential equations into nonlinear ordinary differential equations. The resulting parameter problem and velocity as well as temperature fields are solved using the homotopy 
analysis method (HAM). The analytical solutions reached using HAM are presented and implications discussed.

\section{Mathematical Model}

Consider that the Newtonian fluid flow in a thin liquid film of uniform thickness $h(t)$ on a horizontal thin elastic sheet emerges from a narrow slot at the origin of a Cartesian coordinate system. The fluid motion and heat transfer are due to the stretching of a permeable elastic sheet parallel to the $x$ axis at $y=0$. Two equal and opposite forces are applied along the $x$-axis, keeping the origin fixed. Under these assumptions, the governing conservation equations of mass, momentum, and energy at unsteady state can be expressed as

$$
\begin{gathered}
\frac{\partial u}{\partial x}+\frac{\partial v}{\partial y}=0 \\
\frac{\partial u}{\partial t}+u \frac{\partial u}{\partial x}+v \frac{\partial u}{\partial y}=v \frac{\partial^{2} u}{\partial y^{2}} \\
\rho C_{p}\left(\frac{\partial T}{\partial t}+u \frac{\partial T}{\partial x}+v \frac{\partial T}{\partial y}\right)=\kappa \frac{\partial^{2} T}{\partial y^{2}},
\end{gathered}
$$

where $u$ is the velocity component of the fluid in the $x$ direction is defined as $U=b x /(1-\alpha t), v_{s}$ is the mass transfer velocity at the surface of the sheet with $v_{s}>0$ for blowing (injection) and $v_{s}<0$ for suction, and $v_{s}=0$ corresponding to an impermeable sheet. Further, $T$ is the temperature, $v$ is the kinematic viscosity, $\rho$ is the density, $C_{p}$ is the specific heat at constant pressure, and $\kappa$ is the thermal diffusivity. It is assumed that the surface of the planar liquid film is smooth and free of surface waves, and the viscous shear stress as well as the heat flux vanishes at the adiabatic free surface. Thus the boundary conditions are

$$
\begin{gathered}
u=U, \quad v=v_{s}, \quad T=T_{s} \quad \text { at } y=0, \\
\frac{\partial u}{\partial y}=\frac{\partial T}{\partial y}=0, \quad v=\frac{d h}{d t} \text { at } y=h .
\end{gathered}
$$

The elastic sheet's temperature is assumed to vary both along the sheet and with time in accordance with

$$
T_{s}=T_{o}-T_{\text {ref }} \frac{b x^{2}}{v}(1-\alpha t)^{-3 / 2},
$$

where $T_{o}$ is the temperature at the slit, $T_{\text {ref }}$ is the constant reference temperature for all $t<1 / \alpha$, and $d$ is the positive constant of proportionality with dimension (length ${ }^{2-r}$ time $^{-1}$ ).

Consider employing the similarity transformations which are given as

$$
\begin{gathered}
\psi=\beta x\left[\frac{v b}{1-\alpha t}\right]^{1 / 2} f(\eta), \\
T=T_{o}-T_{\text {ref }}\left[\frac{b x^{2}}{v(1-\alpha t)^{-3 / 2}}\right] \theta(\eta), \\
\eta=\frac{1}{\beta}\left[\frac{b}{v(1-\alpha t)}\right]^{1 / 2} y,
\end{gathered}
$$

where $\beta$ is the dimensionless film thickness and $\psi(x, y)$ is the stream function defined by

$$
\begin{gathered}
u=\frac{\partial \psi}{\partial y}=\frac{b x}{1-\alpha t} f^{\prime}(\eta), \\
v=-\frac{\partial \psi}{\partial x}=-\left(\frac{v b}{1-\alpha t}\right)^{1 / 2} \beta f(\eta),
\end{gathered}
$$

where a prime denotes differentiation with respect to $\eta$. Apparently, (7) has already satisfied the continuity equation, (1). Consequently, (1)-(4) are transformed to the following nonlinear boundary-value problem:

$$
\begin{aligned}
& f^{\prime \prime \prime}+\gamma\left(f f^{\prime \prime}-\frac{1}{2} S \eta f^{\prime \prime}-\left(f^{\prime}\right)^{2}-S f^{\prime}\right)=0, \\
& \frac{1}{\operatorname{Pr}} \theta^{\prime \prime}+\gamma\left(f \theta^{\prime}-2 f^{\prime} \theta-\frac{1}{2} S \eta \theta^{\prime}-\frac{3}{2} S \theta\right)=0,
\end{aligned}
$$

subject to

$$
\begin{aligned}
f(0) & =K, & f^{\prime}(0) & =1, \\
f(1) & =\frac{1}{2} S, & f^{\prime \prime}(1) & =0, \\
\theta(0) & =1, & \theta^{\prime}(1) & =0,
\end{aligned}
$$

where $S=\alpha / b$ is a dimensionless measure of the unsteadiness, $\gamma=\beta^{2}$ is an unknown constant which must be determined as a part of the boundary value problem, $\operatorname{Pr}=$ $\nu / \kappa$ is Prandtl number, and $K=v_{s} / \beta(\nu b)^{1 / 2}$ is permeability parameter. It is worth mentioning that the momentum boundary layer problem defined by the ODE (8) subject to the relevant boundary conditions (10) is decoupled from the thermal boundary layer problem, while the temperature field $\theta(\eta)$ is on the other hand coupled to the velocity field. The most important characteristics of flow and heat transfer are the shear stress $\tau_{s}$ and the heat flux $q_{s}$ on the stretching sheet that are defined as

$$
\begin{gathered}
\tau_{s}=\mu\left(\frac{\partial u}{\partial y}\right)_{y=0}, \\
q_{s}=-\kappa\left(\frac{\partial T}{\partial y}\right)_{y=0},
\end{gathered}
$$

where $\mu$ is the fluid dynamic viscosity. The local skin-friction coefficient $C_{f}$ and the local Nusselt number $\mathrm{Nu}_{x}$ can be defined as

$$
\begin{aligned}
C_{f} & =\frac{\tau_{s}}{\rho u^{2} / 2}, \\
\mathrm{Nu}_{x} & =-\frac{x q_{s}}{\kappa T_{\mathrm{ref}}} .
\end{aligned}
$$

Thus, the skin friction and the rate of heat transfer for fluid flow in a thin film can be expressed as

$$
\begin{gathered}
\frac{1}{2} C_{f} \operatorname{Re}_{x}^{1 / 2}=\frac{1}{\beta} f^{\prime \prime}(0), \\
\mathrm{Nu}_{x} \operatorname{Re}_{x}^{-1 / 2}=\frac{b x^{2}}{\beta v(1-\alpha t)^{1 / 2}} \theta^{\prime}(0),
\end{gathered}
$$

where $\operatorname{Re}_{x}=U x / v$ is the local Reynolds number. 


\section{Analytical Approach}

In this section we apply HAM to solve systems (8)-(11). We assume that the solutions of $f(\eta)$ and $\theta(\eta)$ can be expressed by a set of base functions $\left\{\eta^{m} \mid m=0,1,2, \ldots\right\}$ with the following forms:

$$
\begin{aligned}
& f(\eta)=\sum_{m=0}^{+\infty} a_{m} \eta^{m}, \\
& \theta(\eta)=\sum_{m=0}^{+\infty} c_{m} \eta^{m},
\end{aligned}
$$

where $a_{m}$ and $c_{m}$ are constants. Under the rule of solution expression given by (8) and (9), subject to the boundary conditions (10) and (11), it is straightforward to choose

$$
\begin{gathered}
f_{0}(\eta)=\left(\frac{-S}{4}+\frac{K}{2}+\frac{1}{2}\right) \eta^{3}+\left(\frac{3 S}{4}-\frac{3 K}{2}-\frac{3}{2}\right) \eta^{2}+\eta+K \\
\theta_{0}(\eta)=1
\end{gathered}
$$

as the initial guesses of $f(\eta)$ and $\theta(\eta)$. The auxiliary linear operators $L_{f}=\partial^{3} / \partial \eta^{3}$ and $L_{\theta}=\partial^{2} / \partial \eta^{2}$ are chosen with the following properties:

$$
\begin{gathered}
L_{f}\left[C_{1}+C_{2} \eta+C_{3} \eta^{2}\right]=0, \\
L_{\theta}\left[C_{4}+C_{5} \eta\right]=0,
\end{gathered}
$$

where $C_{1}, C_{2}, C_{3}, C_{4}$, and $C_{5}$ are constants of integration. From (8) and (9), the nonlinear operators are defined as

$$
\begin{aligned}
& N_{f}[F(\eta, q), \Gamma(q)] \\
& =F^{\prime \prime \prime}+\Gamma\left[F F^{\prime \prime}-\frac{1}{2} S \eta F^{\prime \prime}-\left(F^{\prime}\right)^{2}-S F^{\prime}\right], \\
& N_{\theta}[F(\eta, q), \Theta(\eta, q), \Gamma(q)] \\
& \quad=\frac{1}{\operatorname{Pr}} \Theta^{\prime \prime}+\Gamma\left[F \Theta^{\prime}-2 F^{\prime} \Theta-\frac{1}{2} S \eta \Theta^{\prime}-\frac{3}{2} S \Theta\right],
\end{aligned}
$$

where $F(\eta, q)$ and $\Theta(\eta, q)$ are both unknown functions of $\eta$ and $q$ while $\Gamma$ is a function dependent on the embedding parameter $q$. Let $\hbar_{f}$ and $\hbar_{\theta}$ denote the non-zero auxiliary parameters whereas $H_{f}$ and $H_{\theta}$ denote nonzero auxiliary functions, respectively. The zero-order deformation equation can be constructed as

$$
\begin{aligned}
& (1-q) L_{f}\left[F(\eta, q)-f_{0}(\eta)\right] \\
& =q \hbar_{f} H_{f} N_{f}[F(\eta, q), \Gamma(q)], \\
& (1-q) L_{\theta}\left[\Theta(\eta, q)-\theta_{0}(\eta)\right] \\
& =q \hbar_{\theta} H_{\theta} N_{\theta}[F(\eta, q), \Theta(\eta, q), \Gamma(q)],
\end{aligned}
$$

subject to the boundary conditions

$$
\begin{array}{lll}
F(0, q)=K, & F^{\prime}(0, q)=1, & \Theta(0, q)=1, \\
F(1, q)=\frac{1}{2} S, & F^{\prime \prime}(1, q)=0, & \Theta^{\prime}(1, q)=0 .
\end{array}
$$

From (17) and (18), it is straightforward to show that when $q=0$, the solutions of (21)-(22) are

$$
F(\eta, 0)=f_{0}(\eta), \quad \Theta(\eta, 0)=\theta_{0}(\eta) .
$$

Since $\hbar_{f}, \hbar_{\theta} \neq 0$ and $H_{f}, H_{\theta} \neq 0$ when $q=1,(21)$-(22) are equivalent to (8)-(11), respectively, provided that

$$
F(\eta, 1)=f(\eta), \quad \Theta(\eta, 1)=\theta(\eta), \quad \Gamma(1)=\gamma .
$$

Thus, as $q$ increases from 0 to $1, F(\eta, q)$ and $\Theta(\eta, q)$ vary from the initial guesses $f_{0}(\eta)$ and $\theta_{0}(\eta)$ to the solutions $f(\eta)$ and $\theta(\eta)$ in (8)-(11). In addition, $\Gamma$ also varies from the initial guess

$$
\Gamma(0)=\gamma_{0}
$$

to $\gamma$. Applying the Taylor series to (23) and (25), $F(\eta, q)$, $\Theta(\eta, q)$, and $\Gamma(q)$ can be expanded as series of $q$

$$
\begin{gathered}
F(\eta, q)=f_{0}(\eta)+\sum_{m=1}^{+\infty} f_{m}(\eta) q^{m}, \\
\Theta(\eta, q)=\Theta_{0}(\eta)+\sum_{m=1}^{+\infty} \theta_{m}(\eta) q^{m}, \\
\Gamma(q)=\gamma_{0}+\sum_{m=1}^{+\infty} \gamma_{m} q^{m},
\end{gathered}
$$

where

$$
\begin{aligned}
f_{m}(\eta) & =\frac{1}{m !}\left[\frac{\partial^{m} F(\eta, q)}{\partial q^{m}}\right]_{q=0}, \\
\theta_{m}(\eta) & =\frac{1}{m !}\left[\frac{\partial^{m} \Theta(\eta, q)}{\partial q^{m}}\right]_{q=0}, \\
\gamma_{m} & =\frac{1}{m !}\left[\frac{\partial^{m} \Gamma(q)}{\partial q^{m}}\right]_{q=0} .
\end{aligned}
$$

Thus, using (24), we have

$$
\begin{gathered}
f(\eta)=f_{0}(\eta)+\sum_{m=1}^{+\infty} f_{m}(\eta), \\
\theta(\eta)=\theta_{0}(\eta)+\sum_{m=1}^{+\infty} \theta_{m}(\eta), \\
\gamma=\gamma_{0}+\sum_{m=1}^{+\infty} \gamma_{m} .
\end{gathered}
$$

By differentiating (21) $m$ times with respect to $q$, then setting $q=0$, and finally dividing by $m$ !, the $m$ th-order deformation equations are obtained:

$$
\begin{aligned}
& L_{f}\left[f_{m}(\eta)-\chi_{m} f_{m-1}(\eta)\right]=\hbar_{f} H_{f}(\eta) R_{1, m}(\eta), \\
& L_{\theta}\left[\theta_{m}(\eta)-\chi_{m} \theta_{m-1}(\eta)\right]=\hbar_{\theta} H_{\theta}(\eta) R_{2, m}(\eta),
\end{aligned}
$$


subject to the boundary conditions

$$
\begin{array}{lll}
f_{m}(0)=K, & f_{m}^{\prime}(0)=0, & \theta_{m}(0)=0, \\
f_{m}(1)=0, & f_{m}^{\prime \prime}(1)=0, & \theta_{m}^{\prime}(1)=0,
\end{array}
$$

for $m \geq 1$, where

$$
\begin{gathered}
R_{1, m}(\eta)=f_{m-1}^{\prime \prime \prime}+\sum_{n=0}^{m-1} \gamma_{m-1-n} \sum_{i=0}^{n}\left(f_{i} f_{n-i}^{\prime \prime}-f_{i}^{\prime} f_{n-i}^{\prime}\right) \\
-\frac{1}{2} S \eta \sum_{n=0}^{m-1} \gamma_{n} f_{m-1-n}^{\prime \prime}-S \sum_{n=0}^{m-1} \gamma_{n} f_{m-1-n}^{\prime}, \\
R_{2, m}(\eta)=\frac{1}{\operatorname{Pr}} \theta_{m-1}^{\prime \prime}+\sum_{n=0}^{m-1} \gamma_{m-1-n} \sum_{i=0}^{n}\left(f_{i} \theta_{n-i}^{\prime}-2 f_{n-i}^{\prime} \theta_{i}\right) \\
-\frac{1}{2} S \eta \sum_{n=0}^{m-1} \gamma_{n} \theta_{m-1-n}^{\prime}-\frac{3}{2} S \sum_{n=0}^{m-1} \gamma_{n} \theta_{m-1-n}, \\
\chi_{m}= \begin{cases}1 & m>1, \\
0 & m=1 .\end{cases}
\end{gathered}
$$

Let $f_{m}^{*}$ and $\theta_{m}^{*}$ denote the particular solutions of (29) as

$$
\begin{gathered}
f_{m}^{*}=\int_{0}^{\eta} \int_{0}^{\eta} \int_{0}^{\eta} \hbar_{f} H_{f}(s) R_{1, m}(s) d s d \eta d \eta+\chi_{m} f_{m-1}, \\
\theta_{m}^{*}=\int_{0}^{\eta} \int_{0}^{\eta} \hbar_{\theta} H_{\theta}(s) R_{2, m}(s) d s d \eta+\chi_{m} \theta_{m-1} .
\end{gathered}
$$

The general solutions for (19) are

$$
\begin{gathered}
f_{m}(\eta)=f_{m}^{*}+C_{1}+C_{2} \eta+C_{3} \eta^{2}, \\
\theta_{m}(\eta)=\theta_{m}^{*}+C_{4}+C_{5} \eta .
\end{gathered}
$$

Hence, the $m$ th-order approximations of $f(\eta), \theta(\eta)$, and $\gamma$ are given, respectively, by

$$
\begin{aligned}
f(\eta) & \approx \sum_{n=0}^{m} f_{n}(\eta), \\
\theta(\eta) & \approx \sum_{n=0}^{m} \theta_{n}(\eta), \\
\gamma & \approx \sum_{n=0}^{m-1} \gamma_{n} .
\end{aligned}
$$

\section{Results and Discussion}

We solve (29)-(30) with the aid of Maple, a symbolic computation software. The auxiliary functions $\hbar_{f}$ and $\hbar_{\theta}$ in (29) were set to be equal to 1 in all calculations reported in this paper. The solution of (29)-(30) can be expressed as

$$
f_{m}(\eta)=\sum_{k=2}^{4 m+3} a_{m, k}(\eta) \eta^{k}, \quad \theta_{m}(\eta)=\sum_{k=1}^{4 m} c_{m, k}(\eta) \eta^{k}
$$

for $m \geq 1$, where $a_{m, k}$ and $c_{m, k}$ are the coefficients, which can be obtained recursively for $m=1,2,3, \ldots$ using

$$
\begin{aligned}
& a_{0,1}=1, \quad a_{0,2}=\frac{3 S}{4}-\frac{3 K}{2}-\frac{3}{2}, \\
& a_{0,3}=\frac{-S}{4}+\frac{K}{2}+\frac{1}{2}, \quad b_{0,0}=1,
\end{aligned}
$$

given by (16) and (17). When $m=1$, we have the analytic solutions as follows:

$$
\begin{gathered}
f_{1}(\eta)=\sum_{k=2}^{7} a_{1, k}(\eta) \eta^{k}, \quad \theta_{1}(\eta)=\sum_{k=1}^{4} c_{1, k}(\eta) \eta^{k}, \\
\gamma_{0}=\frac{105\left(2-S+2 K-2 K / \hbar_{f}\right)}{R},
\end{gathered}
$$

where

$$
\begin{aligned}
& a_{1,2}=\frac{-3}{8 R}\left(152 K \hbar_{f} S+76 \hbar_{f} K^{2} S-94 \hbar_{f} K S^{2}-112 K+24 \hbar_{f}\right. \\
& -504 K^{2}+72 \hbar_{f} K+76 \hbar_{f} S+112 K S-94 \hbar_{f} S^{2} \\
& +24 \hbar_{f} K^{3}-238 K S^{2}+812 K^{2} S+72 \hbar_{f} K^{2} \\
& \left.+25 S^{3} \hbar_{f}-672 K^{3}\right) \\
& a_{1,3}=\frac{-1}{2 R}\left(-210 K^{3}-210 K^{2}-70 K+96 \hbar_{f} S+144 \hbar_{f} K\right. \\
& -70 K S-132 \hbar_{f} S^{2} K+96 K^{2} \hbar_{f} S+48 \hbar_{f} \\
& +144 K^{2} \hbar_{f}+48 K^{3} \hbar_{f}-132 \hbar_{f} S^{2}+36 \hbar_{f} S^{3} \\
& \left.+105 K^{2} S+192 S \hbar_{f} K\right) \\
& a_{1,4}=\frac{105}{32 R}\left(2 \hbar_{f}-\hbar_{f} S+2 \hbar_{f} K-2 K\right) \\
& \times\left(4 K^{2}+8 K-3 S^{2}+4 S+4 K S+4\right), \\
& a_{1,5}=\frac{-21}{32 R}\left(2 \hbar_{f}-\hbar_{f} S+2 \hbar_{f} K-2 K\right) \\
& \times\left(24 K+12 K^{2}-4 S-S^{2}-4 K S+12\right), \\
& a_{1,6}=\frac{21}{32 R}\left(2 \hbar_{f}-\hbar_{f} S+2 \hbar_{f} K-2 K\right) \\
& \times\left(4 K^{2}+8 K+S^{2}-4 S+4-4 K S\right), \\
& a_{1,7}=\frac{-3}{32 R}\left(2 \hbar_{f}-\hbar_{f} S+2 \hbar_{f} K-2 K\right) \\
& \times\left(4 K^{2}+8 K+S^{2}-4 S+4-4 K S\right),
\end{aligned}
$$




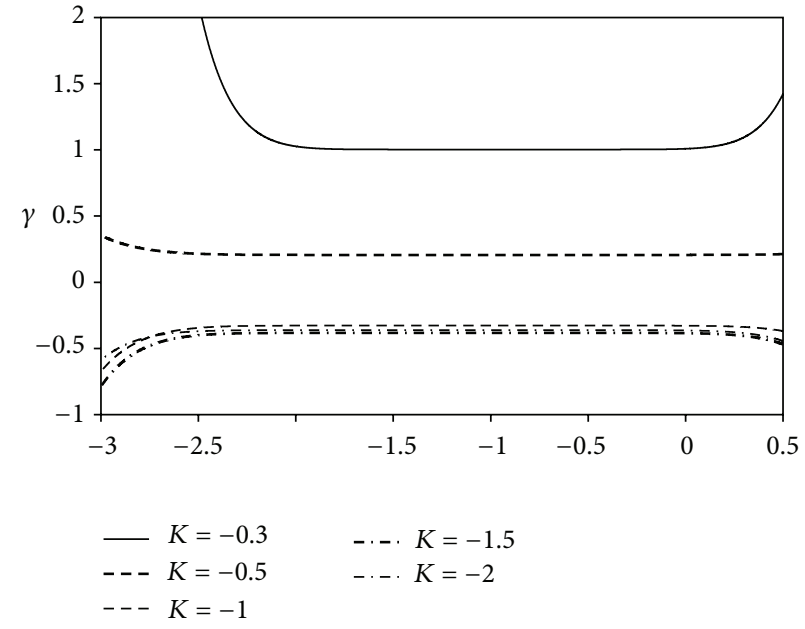

(a) Suction

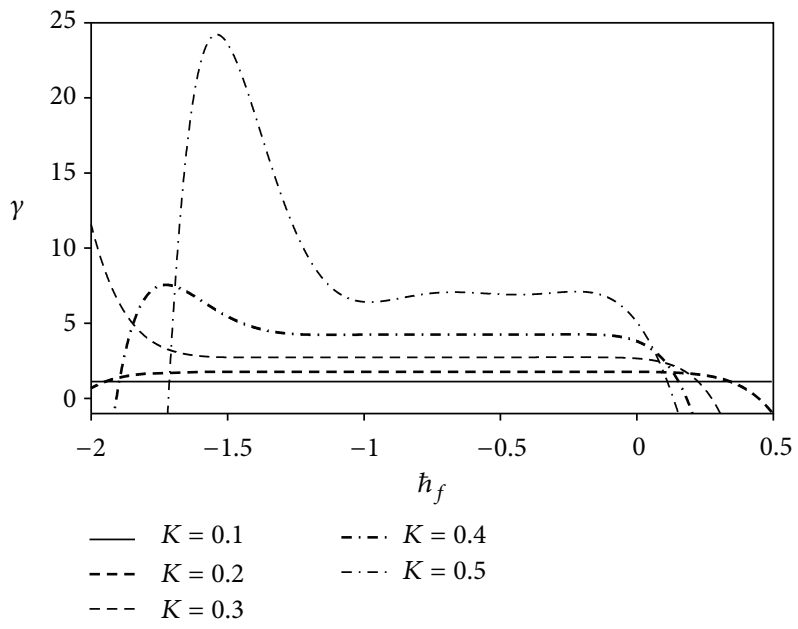

(b) Blowing

FIGURE 1: The $\hbar_{f}$ curve of $\gamma=\beta^{2}$ using 10th-order HAM approximation for the cases $S=0.8$ and $\operatorname{Pr}=1$.

$$
\begin{aligned}
& c_{1,1}=\frac{-105}{2 R} \frac{\hbar_{\theta}}{\hbar_{f}}\left(2 \hbar_{f}-\hbar_{f} S+2 \hbar_{f} K-2 K\right)(4 K-5 S), \\
& c_{1,2}=\frac{-105}{4 R} \frac{\hbar_{\theta}}{\hbar_{f}}\left(2 \hbar_{f}-\hbar_{f} S+2 \hbar_{f} K-2 K\right)(4+3 S), \\
& c_{1,3}=\frac{105}{2 R} \frac{\hbar_{\theta}}{\hbar_{f}}\left(2 \hbar_{f}-\hbar_{f} S+2 \hbar_{f} K-2 K\right)(2 K-S+2), \\
& c_{1,4}=\frac{-105}{8 R} \frac{\hbar_{\theta}}{\hbar_{f}}\left(2 \hbar_{f}-\hbar_{f} S+2 \hbar_{f} K-2 K\right)(2 K-S+2),
\end{aligned}
$$

where $R=162 K^{2}-225 K S+114 K+72 S^{2}-50 S+22$. Note that (37) will be simplified to the expression $\gamma_{0}$ of Wang [4] when $K=0$.

We noted that the HAM analytic solutions contain two nonzero auxiliary parameters $\hbar_{f}$ and $\hbar_{\theta}$ that can be used to adjust and control the convergence of the series solutions. Liao $[15,25]$ indicates that the analytic solutions given by HAM contain an auxiliary parameter $\hbar$ which could be chosen by means of what he calls the $\hbar$-curve.

Figures 1(a) and 1(b) show the variation of $\gamma$ with $\hbar_{f}$ using 10th-order HAM approximation when $S=0.8$ for suction and $S=1.4$ for blowing, respectively. For suction case, it is shown that the convergence region can be obtained by choosing a value of $\hbar_{f}$ in the ranges $-1.8 \leq \hbar_{f} \leq-0.1$ for $K=-0.3$, $-2.5 \leq \hbar_{f} \leq 0.3$ for $K=-0.5,-2.3 \leq \hbar_{f} \leq-0.2$ for $K=-1$, $-2.3 \leq \hbar_{f} \leq-0.5$ for $K=-1.5$, and $-2.3 \leq \hbar_{f} \leq-0.5$ for $K=-2$.

For blowing case, the appropriate ranges of the auxiliary parameter $\hbar_{f}$ to maintain the convergent rate and region of $\gamma$ are $-2 \leq \hbar_{f} \leq-0.5$ for $K=0.1,-1.75 \leq \hbar_{f} \leq 0$ for $K=0.2$, $-1.4 \leq \hbar_{f} \leq-0.1$ for $K=0.3,-1.2 \leq \hbar_{f} \leq-0.1$ for $K=0.4$, and $-0.65 \leq \hbar_{f} \leq-0.5$ for $K=0.5$.

The $\hbar_{f}$ curves of $f^{\prime \prime}(0)$ are graphed in Figures 2(a) and 2(b) for suction and blowing, respectively. For suction case, it is shown that the convergence region can be obtained by choosing a value of $\hbar_{f}$ in the ranges $-1.5 \leq \hbar_{f} \leq-0.4$ for $K=-0.3,-1.8 \leq \hbar_{f} \leq 0$ for $K=-0.5,-1.9 \leq \hbar_{f} \leq-0.1$ for $K=-1,-1.7 \leq \hbar_{f} \leq-0.3$ for $K=-1.5$, and $-1.5 \leq \hbar_{f} \leq$ -0.5 for $K=-2$.

For blowing case, the appropriate ranges of the auxiliary parameter $\hbar_{f}$ to maintain the convergent rate and region of $f^{\prime \prime}(0)$ are $-1.5 \leq \hbar_{f} \leq-0.25$ for $K=0.1,-1.4 \leq \hbar_{f} \leq-0.4$ for $K=0.2,-1.2 \leq \hbar_{f} \leq-0.5$ for $K=0.3,-1 \leq \hbar_{f} \leq-0.5$ for $K=0.4$, and $-0.55 \leq \hbar_{f} \leq-0.45$ for $K=0.5$.

In order to retain the convergent rate and region of $\theta^{\prime}(0)$, Figures 3(a) and 3(b) show the appropriate ranges of $\hbar_{\theta}$ for both suction and blowing. For suction case, the appropriate value of $\hbar_{\theta}$ is in the ranges $-0.8 \leq \hbar_{\theta} \leq-0.6$ for $K=-0.3$, $-1.4 \leq \hbar_{\theta} \leq-0.4$ for $K=-0.5,-1.75 \leq \hbar_{\theta} \leq-0.75$ for $K=-0.8$, and $-1.5 \leq \hbar_{\theta} \leq-1.5$ for $K=-1$.

For blowing case, the appropriate value of $\hbar_{\theta}$ is in the ranges $-1 \leq \hbar_{\theta} \leq-0.4$ for $K=0.1,-0.75 \leq \hbar_{\theta} \leq-0.5$ for $K=0.2,-0.6 \leq \hbar_{\theta} \leq-0.4$ for $K=0.3,-0.5 \leq \hbar_{\theta} \leq-0.45$ for $K=0.4$, and $-0.55 \leq \hbar_{\theta} \leq-0.5$ for $K=0.5$.

To demonstrate the accuracy of the present findings, results for dimensionless film thickness and the skin friction are compared with the available results obtained by Wang [4]. It is found that all the numerical results in Table 1 agree with the values of $\beta$ and $-f^{\prime \prime}(0)$ in Wang [4]. As the unsteadiness parameter $S$ increases, the skin friction $-f^{\prime \prime}(0)$ increases and thereby the film thickness reduces.

The effects of permeability parameter $K$ on the velocity distribution $f^{\prime}(\eta)$ are presented in Figures $4(\mathrm{a})$ and $4(\mathrm{~b})$ for suction and blowing, respectively. It is found that, as permeability parameter $K$ increases, the flow velocity of the thin film increases for suction case, while for blowing case, the flow velocity decreases.

Figures 5(a) and 5(b) reveal that by increasing the values of unsteadiness parameter, the velocity of thin film flow increases for both cases of suction $(K<0)$ and blowing $(K>0)$. 


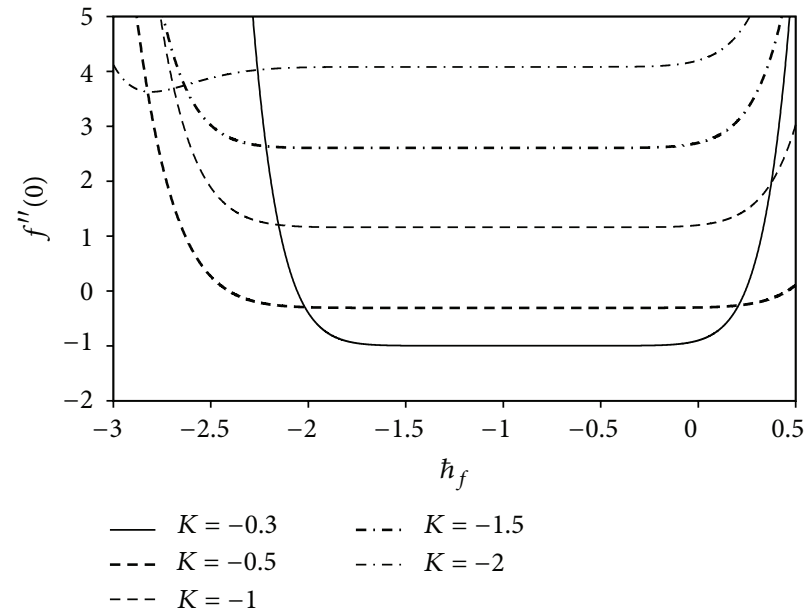

(a) Suction

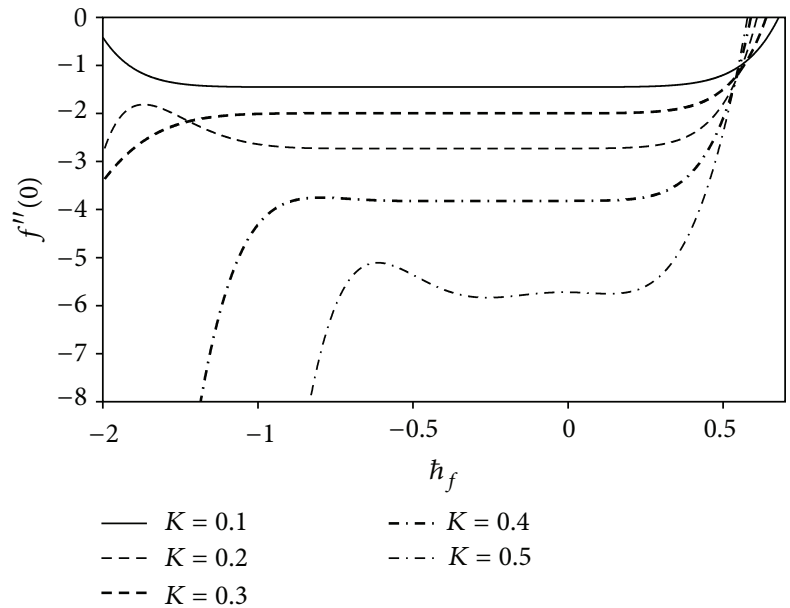

(b) Blowing

FIgURE 2: The $\hbar_{f}$ curve of $f^{\prime \prime}(0)$ using 10th-order HAM approximation for the cases $S=0.8$ and $\operatorname{Pr}=1$.

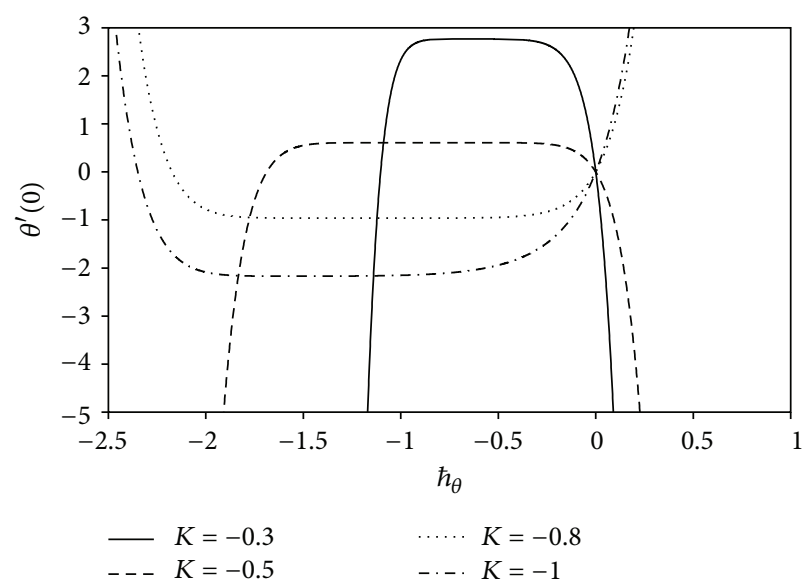

(a) Suction

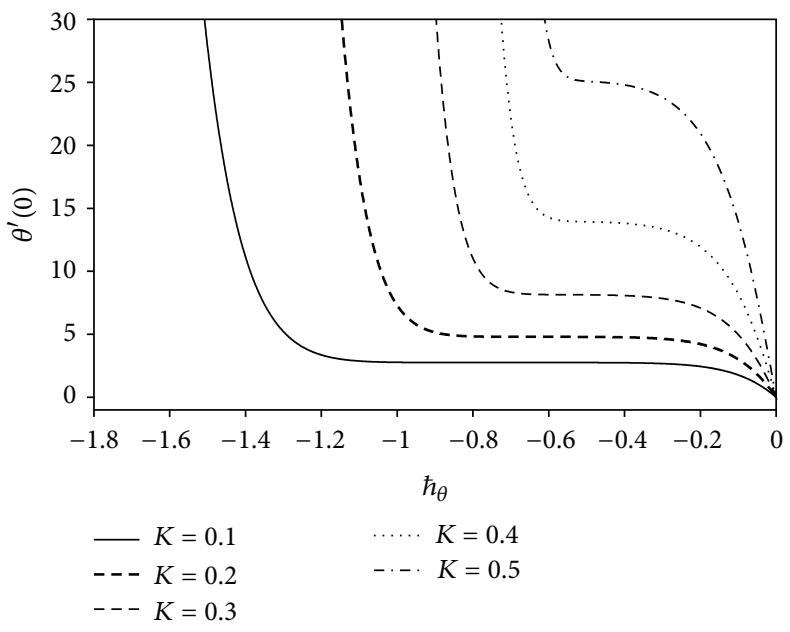

(b) Blowing

FIGURE 3: The $\hbar_{\theta}$ curve of $\theta^{\prime}(0)$ using 10th-order HAM approximation for the cases $S=0.8, \operatorname{Pr}=1$, and $\hbar_{f}=-0.8$.

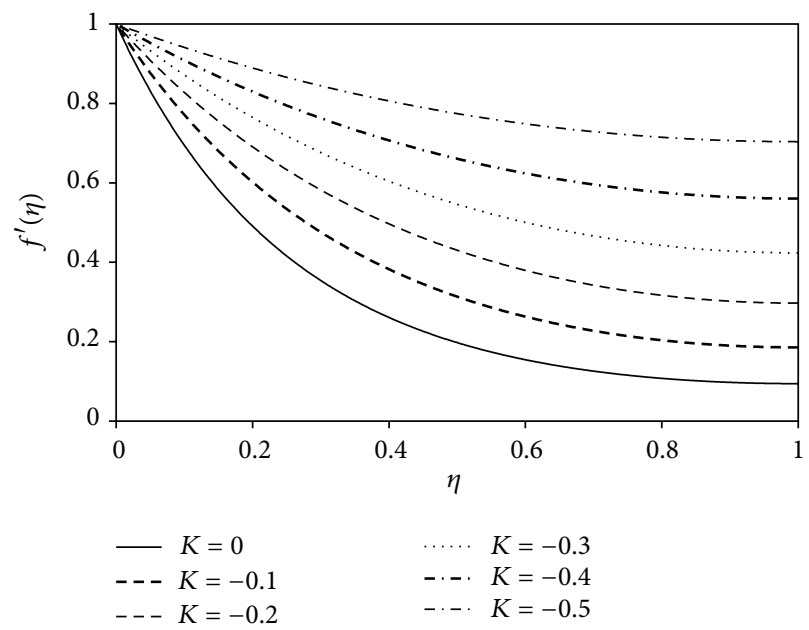

(a) Suction

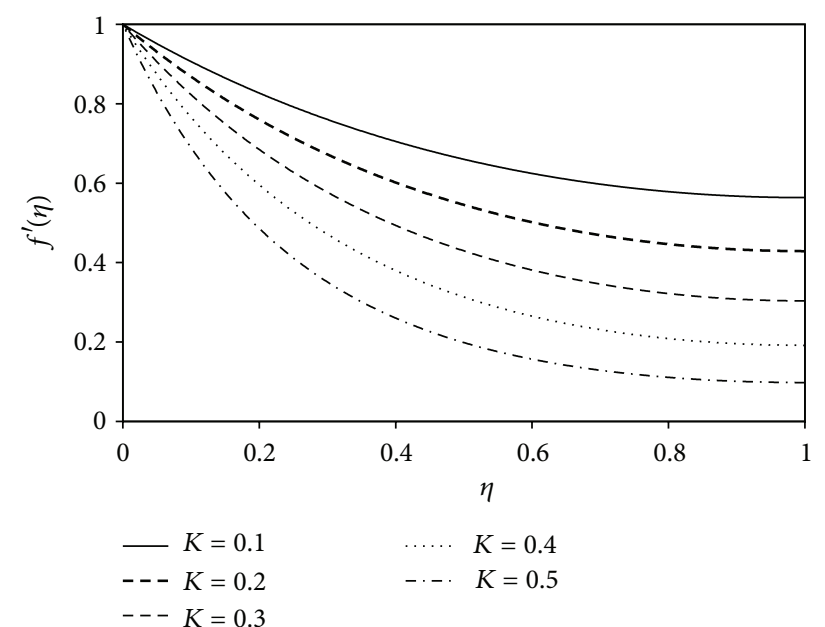

(b) Blowing

FIgURE 4: The effect of permeability parameter $K$ on the velocity distributions $f^{\prime}(\eta)$ using 10th-order HAM approximation when $\operatorname{Pr}=1$. 


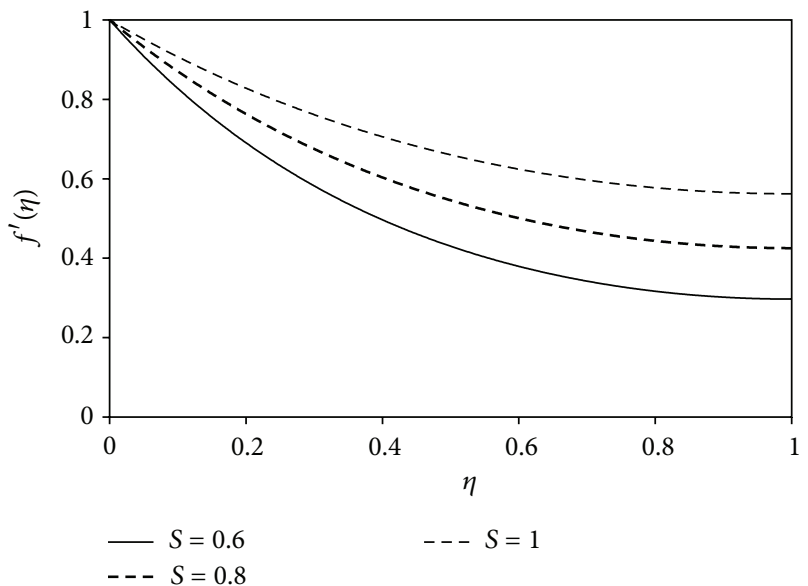

(a) Suction $(K=-0.2)$

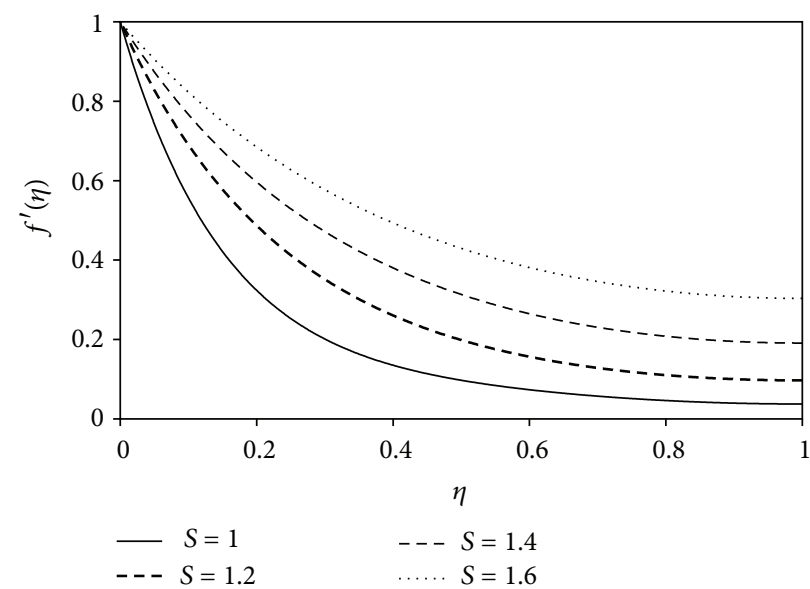

(b) Blowing $(K=0.3)$

FIGURE 5: The effect of unsteadiness parameter $S$ on the velocity distributions $f^{\prime}(\eta)$ using 10th-order HAM approximation when Pr $=1$.

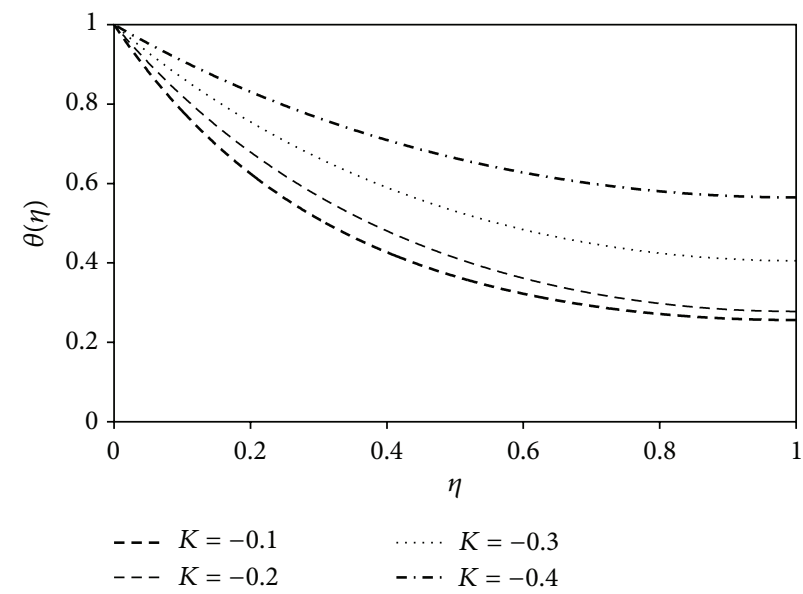

(a) Suction

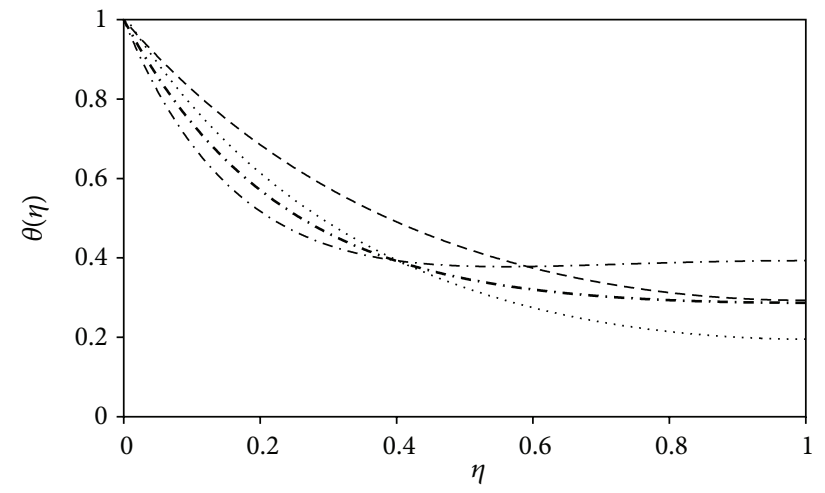

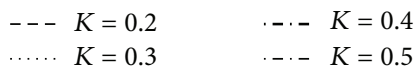

(b) Blowing

FIGURE 6: The effect of permeability parameter $K$ on the temperature distributions $\theta(\eta)$ using 10th-order HAM approximation when Pr $=1$.

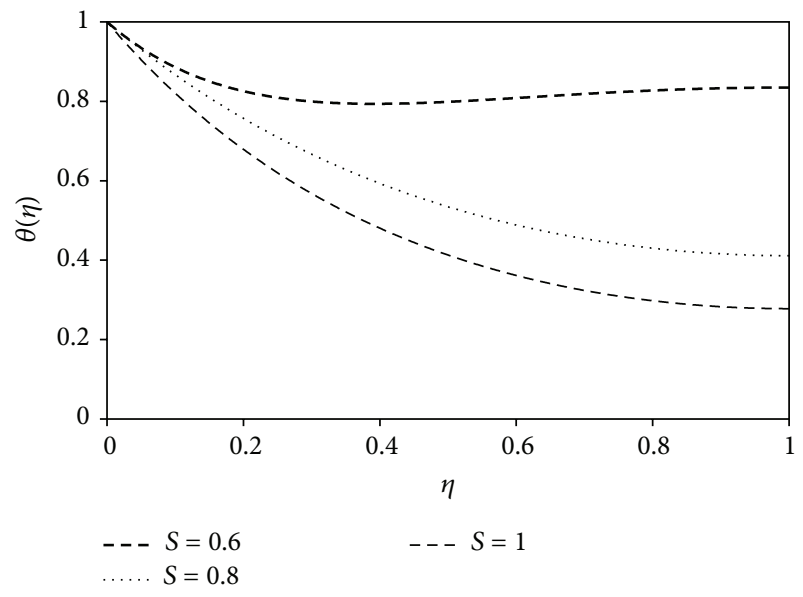

(a) Suction $(K=-0.2)$

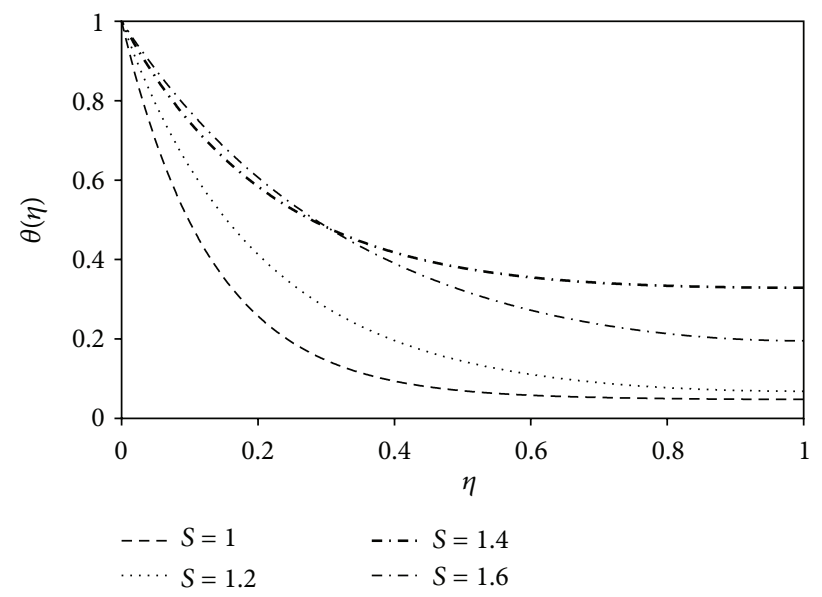

(b) Blowing $(K=0.3)$

FIGURE 7: The effect of unsteadiness parameter $S$ on the temperature distributions $\theta(\eta)$ using 10th-order HAM approximation when $\operatorname{Pr}=1$. 
TABLE 1: Variations of the dimensionless film thickness $\beta=\gamma^{1 / 2}$ and the skin friction $-f^{\prime \prime}(0)$ with respect to the unsteadiness parameter when $\operatorname{Pr}=1$ and $K=0$ using 10th-order HAM approximation.

\begin{tabular}{lccccc}
\hline & \multicolumn{2}{c}{ Present work } & \multicolumn{2}{c}{ Wang [4] } \\
$S$ & $\hbar_{f}$ & $\beta$ & $-f^{\prime \prime}(0)$ & $\beta$ & $-f^{\prime \prime}(0)$ \\
\hline 0.8 & -0.6 & 2.151994 & 2.680943 & 2.15199 & 2.68094 \\
1.0 & -0.8 & 1.543616 & 1.972384 & 1.54362 & 1.97238 \\
1.2 & -1.0 & 1.127780 & 1.442625 & 1.127780 & 1.442631 \\
1.4 & -1.0 & 0.821032 & 1.012784 & 0.821032 & 1.012784 \\
1.6 & -0.6 & 0.576173 & 0.642397 & 0.567173 & 0.642397 \\
1.8 & -1.0 & 0.356389 & 0.309137 & 0.356389 & 0.309137 \\
\hline
\end{tabular}

The temperature profiles for different values of suction and blowing are demonstrated in Figures 6(a) and 6(b). It is seen that the temperature of the fluid flow increases with the increase in suction whereas blowing $(K>0)$ has quite the opposite effect on the temperature of the flow.

The effects of the unsteadiness parameter on the temperature profile are shown graphically in Figures 7(a) and 7(b). It is observed that by increasing the value of unsteadiness parameter, the temperature profiles decrease for both cases of suction and blowing.

\section{Concluding Remarks}

In this paper an analysis has been carried out to study the effects of porous stretching sheet for both cases of suction and blowing. Analytical and numerical solutions are obtained for momentum and heat transfer over a permeable stretching sheet. The effect of permeability parameter $K$ is to increase the flow velocity $f^{\prime}(\eta)$ for suction case and to decrease the flow velocity for the blowing case. Besides, we found that the permeability parameter $K$ has significant effects on the temperature distribution. When $K$ increases, the temperature of the fluid flow $\theta(\eta)$ increases for suction case and decreases for blowing case. When the unsteadiness parameter increases, the velocity of thin film flow increases for both cases of suction $(K<0)$ and blowing $(K>0)$; thereby the film thickness reduces.

\section{Acknowledgment}

The authors would like to acknowledge the financial support received from the Grant UKM-ST-07-FRGS0028-2009.

\section{References}

[1] L. J. Crane, "Flow past a stretching plate," Journal of Applied Mathematics and Physics, vol. 21, pp. 645-647, 1970.

[2] C. Y. Wang, "Liquid film on an unsteady stretching surface," Quarterly of Applied Mathematics, vol. 48, no. 4, pp. 601-610, 1990.

[3] H. I. Andersson, J. B. Aarseth, and B. S. Dandapat, "Heat transfer in a liquid film on an unsteady stretching surface," International Journal of Heat and Mass Transfer, vol. 43, no. 1, pp. 69-74, 2000.
[4] C. Wang, "Analytic solutions for a liquid film on an unsteady stretching surface," Heat and Mass Transfer, vol. 42, no. 8, pp. 759-766, 2006.

[5] H. I. Andersson, J. B. Aarseth, N. Braud, and B. S. Dandapat, "Flow of a power-law fluid film on an unsteady stretching surface," Journal of Non-Newtonian Fluid Mechanics, vol. 62, no. 1, pp. 1-8, 1996.

[6] C. H. Chen, "Heat transfer in a power-law fluid film over a unsteady stretching sheet," Heat and Mass Transfer, vol. 39, no. 8-9, pp. 791-796, 2003.

[7] C. H. Chen, "Effect of viscous dissipation on heat transfer in a non-Newtonian liquid film over an unsteady stretching sheet," Journal of Non-Newtonian Fluid Mechanics, vol. 135, no. 2-3, pp. 128-135, 2006.

[8] C. Wang and I. Pop, "Analysis of the flow of a power-law fluid film on an unsteady stretching surface by means of homotopy analysis method," Journal of Non-Newtonian Fluid Mechanics, vol. 138, no. 2-3, pp. 161-172, 2006.

[9] B. S. Dandapat, B. Santra, and H. I. Andersson, "Thermocapillarity in a liquid film on an unsteady stretching surface," International Journal of Heat and Mass Transfer, vol. 46, no. 16, pp. 3009-3015, 2003.

[10] B. S. Dandapat, B. Santra, and K. Vajravelu, "The effects of variable fluid properties and thermocapillarity on the flow of a thin film on an unsteady stretching sheet," International Journal of Heat and Mass Transfer, vol. 50, no. 5-6, pp. 991-996, 2007.

[11] C. H. Chen, "Marangoni effects on forced convection of powerlaw liquids in a thin film over a stretching surface," Physics Letters A, vol. 370, no. 1, pp. 51-57, 2007.

[12] N. F. M. Noor and I. Hashim, "Thermocapillarity and magnetic field effects in a thin liquid film on an unsteady stretching surface," International Journal of Heat and Mass Transfer, vol. 53, no. 9-10, pp. 2044-2051, 2010.

[13] M. S. Abel, N. Mahesha, and J. Tawade, "Heat transfer in a liquid film over an unsteady stretching surface with viscous dissipation in presence of external magnetic field," Applied Mathematical Modelling, vol. 33, no. 8, pp. 3430-3441, 2009.

[14] N. F. M. Noor, O. Abdulaziz, and I. Hashim, "MHD flow and heat transfer in a thin liquid film on an unsteady stretching sheet by the homotopy analysis method," International Journal for Numerical Methods in Fluids, vol. 63, no. 3, pp. 357-373, 2010.

[15] S. J. Liao, Beyond Perturbation: Introduction to the Homotopy Analysis Method, Chapman \& Hall, Boca Raton, Fla, USA, 2004.

[16] S. Liao, "On the homotopy analysis method for nonlinear problems," Applied Mathematics and Computation, vol. 147, no. 2, pp. 499-513, 2004.

[17] S. Abbasbandy, "The application of homotopy analysis method to nonlinear equations arising in heat transfer," Physics Letters A, vol. 360, no. 1, pp. 109-113, 2006.

[18] T. Hayat and M. Sajid, "Homotopy analysis of MHD boundary layer flow of an upper-convected Maxwell fluid," International Journal of Engineering Science, vol. 45, no. 2-8, pp. 393-401, 2007.

[19] S. Abbasbandy, "Homotopy analysis method for the Kawahara equation," Nonlinear Analysis: Real World Applications, vol. 11, no. 1, pp. 307-312, 2010.

[20] S. Abbasbandy, "Approximate solution for the nonlinear model of diffusion and reaction in porous catalysts by means of the homotopy analysis method," Chemical Engineering Journal, vol. 136, no. 2-3, pp. 144-150, 2008. 
[21] O. Abdulaziz, N. F. M. Noor, and I. Hashim, "Homotopy analysis method for fully developed MHD micropolar fluid flow between vertical porous plates," International Journal for Numerical Methods in Engineering, vol. 78, no. 7, pp. 817-827, 2009.

[22] O. Abdulaziz and I. Hashim, "Fully developed free convection heat and mass transfer of a micropolar fluid between porous vertical plates," Numerical Heat Transfer A, vol. 55, no. 3, pp. 270-288, 2009.

[23] T. Hayat, N. Ahmed, M. Sajid, and S. Asghar, "On the MHD flow of a second grade fluid in a porous channel," Computers \& Mathematics with Applications, vol. 54, no. 3, pp. 407-414, 2007.

[24] S. Abbasbandy and T. Hayat, "Solution of the MHD FalknerSkan flow by homotopy analysis method," Communications in Nonlinear Science and Numerical Simulation, vol. 14, no. 9-10, pp. 3591-3598, 2009.

[25] S. Liao, "Notes on the homotopy analysis method: some definitions and theorems," Communications in Nonlinear Science and Numerical Simulation, vol. 14, no. 4, pp. 983-997, 2009. 


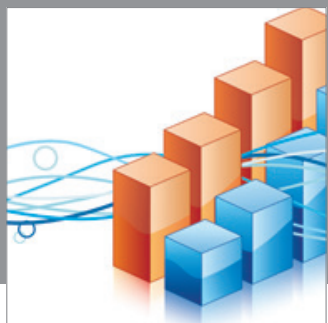

Advances in

Operations Research

mansans

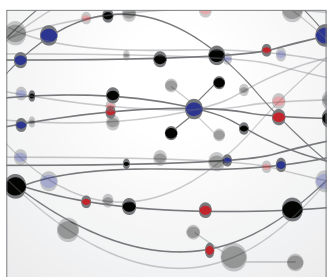

The Scientific World Journal
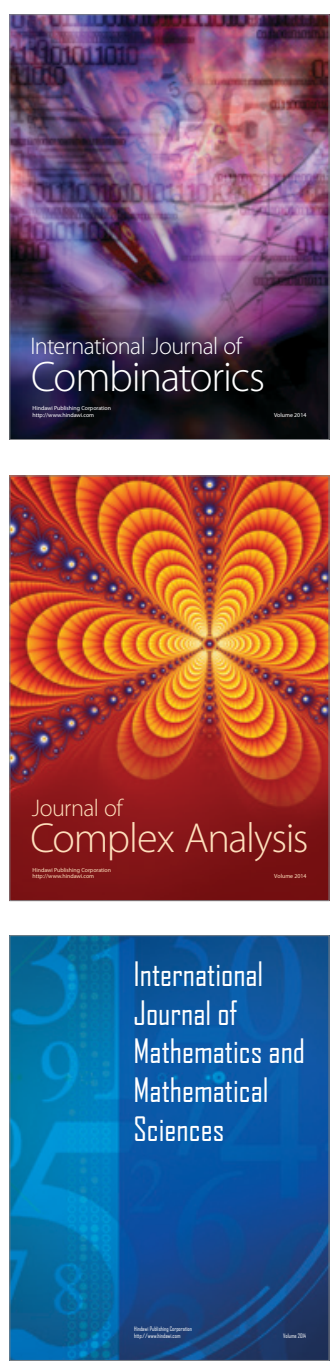
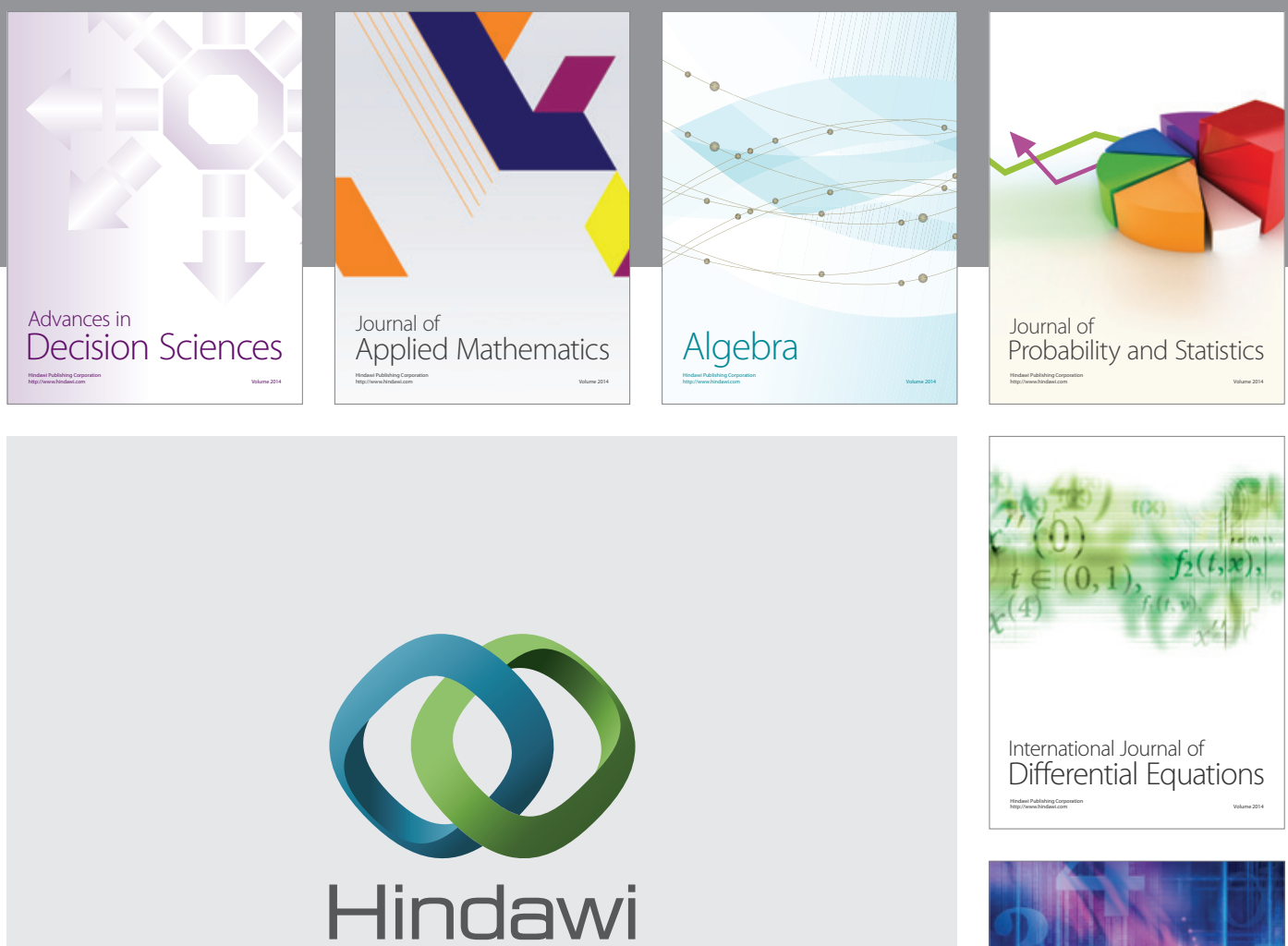

Submit your manuscripts at http://www.hindawi.com
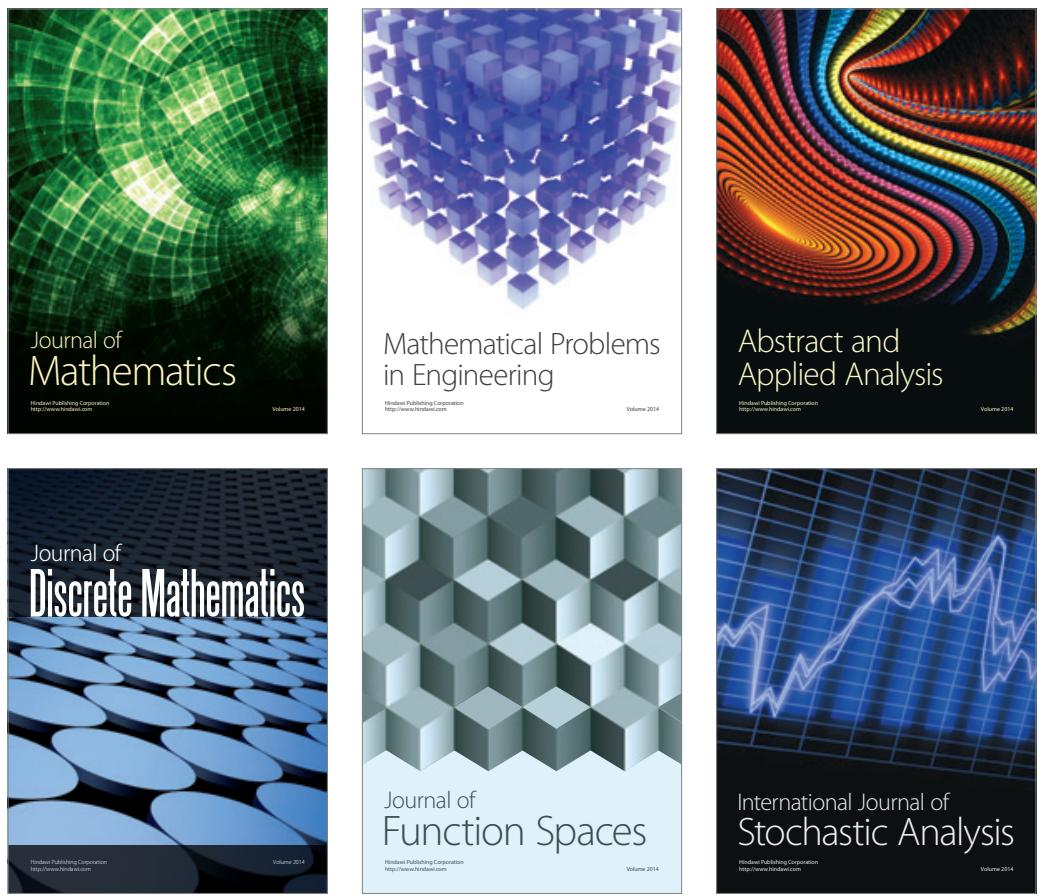

Journal of

Function Spaces

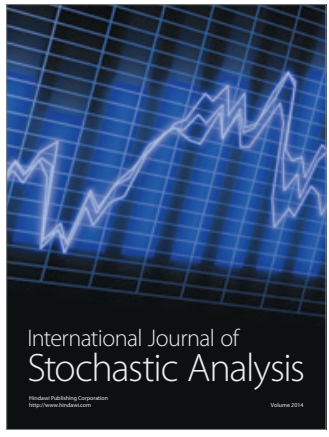

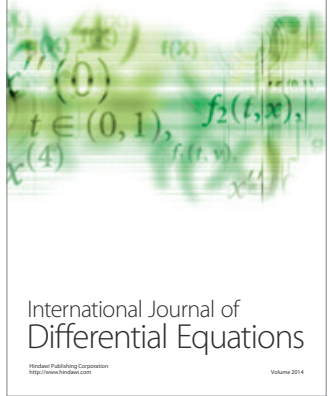
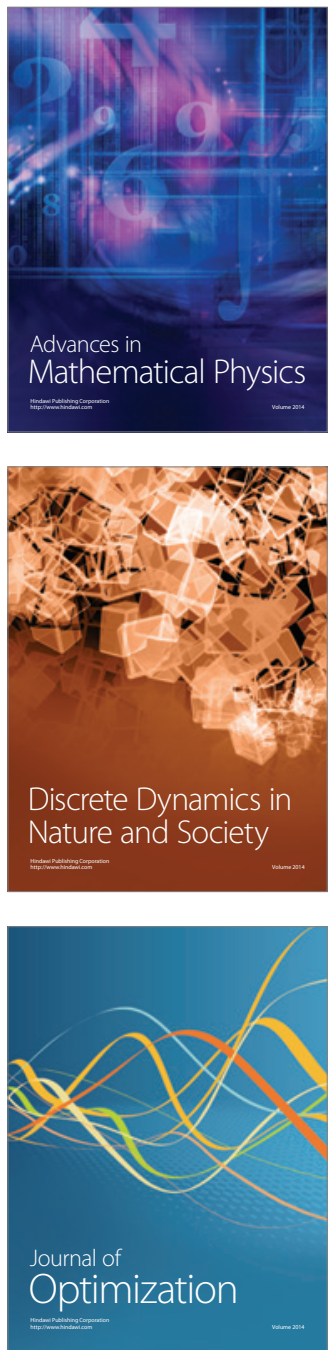